\title{
Can('t) Wait to Have a Robot at Home? - Japanese and German Users' Attitudes Toward Service Robots in Smart Homes
}

\author{
Jasmin Bernotat and Friederike Eyssel, CITEC, Bielefeld University
}

\begin{abstract}
In the present study, we compared Japanese and German participants' perceptions of social robots. To do so, participants from both cultural backgrounds evaluated two types of service robots designed for use in the smart home context. One of the prototypes featured a rather technical appearance, whereas the other prototype featured a newly designed social robot head. Against our predictions, Japanese and German participants evaluated both robot types similarly. Japanese participants generally showed a stronger tendency to anthropomorphize nonhuman agents and attributed more mind, particularly on the experience dimension, to both robot types than German participants. Nonetheless, Japanese and German participants attributed similar levels of agency to both robot types. Unexpectedly, Japanese participants perceived the robots even as less humanlike than German participants. As predicted, Japanese participants reported less attitudinal robot acceptance and tended to show less trust toward both robot types than Germans. Japanese and Germans indicated similar levels of robot anxiety, perceived uncanniness, and robot likeability. Japanese and German participants generally perceived the robots rather as machines and tools than as human companions. Especially German participants wanted to use the robots for rather safe, boring, and simple tasks that do not require any direct interaction with them. Japanese and German participants were not yet familiar with robots and showed less positive attitudes toward them than widely believed (e.g., [1]). Implications how to gain a more realistic view on users' perceptions of robots to enable a fruitful and comfortable HRI are discussed.
\end{abstract}

\section{INTRODUCTION}

Think of claims like: "Everyone likes robots and people can't wait to have them at home." or "Especially Japanese people are crazy about robots." that are often made by developers, marketing managers, and in popular discourse about social robots (e.g., [1]). Indeed, robots are expected to become a part of people's everyday life. But do people really share this enthusiastic view on robots and do Japanese people's attitudes toward robots indeed differ from Europeans' view on robots?

To answer these questions, we conducted a cross-cultural study in Japan and Germany and investigated people's attitudes toward two different types of service robots for use in a smart home. Japan and Germany are amongst the ten countries with the most service robot manufacturers [2]. It is likely that Japanese and Germans will be more and more confronted with robots in their daily life. However, due to cultural differences, it is plausible that Japanese and Germans

\footnotetext{
*This research was supported by the Cluster of Excellence Cognitive Interaction Technology 'CITEC' (EXC 277) at Bielefeld University, which is funded by the German Research Foundation (DFG). It is further related to the European Project CODEFROR (FP7-PIRSES-2013-612555).
}

might perceive robots differently: Shinto and Buddhism are prevalent religions in Japan, while German culture is influenced by Christianity. A Shinto legend holds that Japanese islands were formed by two gods who became progenitors of the gods of Shinto religion and of the first Emperor of Japan [3]. This belief resulted in a deep respect for nature, animals, gods, and heroes. Further, Japanese people believe that a soul, humanlike traits, and intentions are inherent in any entity, such as in gods, heroes, animals, and nature. This is also reflected in Japanese popular culture in which various kinds of nonhuman agents, including robots, are presented as having humanlike traits [4]. According to [5], anthropomorphism, the tendency to ascribe human traits to nonhuman agents, helps to understand others' behavior and thus might reduce uncertainty. Accordingly, one might assume that the tendency to anthropomorphize nonhuman agents and the prevalence of robots in Japanese popular culture is linked to more positive evaluations of HRI and less anxiety toward robots than in Western countries. However, previous studies yielded contradictory findings. For instance, [6] found that Japanese participants rated a social robot higher on animacy, intelligence, and safety than Australian participants. In contrast, [7] have demonstrated that Japanese participants were more concerned about the impact robots might have on society than Chinese and Dutch participants (see [8] for confirming results). Relatedly, [9] have reported that Japanese participants associated less psychological safety, i.e., perceived comfort, with a robot with anthropomorphic features than U.S. Americans. Moreover, Japanese participants associated less controllability and performance with the robot than U.S. Americans. Presenting highly anthropomorphic robots and technical robots, [10] found that U.S. Americans liked both robot types more than Japanese participants. The authors concluded that the greater exposure to robots via media might have influenced Japanese people's attitudes toward robots. In Japanese popular culture, robots are often characterized as highly developed humanlike agents. That is why Japanese people might be more aware of both the advantages and the shortcomings of these highly developed robots that might serve humans or threaten them. Consequently, Japanese people might be more concerned about robots and might hold less positive attitudes toward them than people from Western countries (see [7], [8], [10]). Hence, it is not surprising that in a study by [11], Japanese participants showed less trust toward robots than Australian participants.

J. Bernotat is with Center of Excellence Cognitive Interaction Technology, Bielefeld University, Germany (email: jbernotat@cit-ec.unibielefeld.de).

F. Eyssel is with Center of Excellence Cognitive Interaction Technology, Bielefeld University, Germany (email: feyssel@ cit-ec.uni-bielefeld.de). 
So far, most studies have focused on cultural differences in participants' perceptions of robots in general. However, this research did not consider the context in which HRI takes place. To illustrate, service robots are expected to play an increasingly important role within the home context and they will, without doubt, have a strong impact on people's everyday life. Until now, however, service robots are still a novelty and people are not yet familiar with them. To facilitate a pleasant HRI, robots should feature humanlike characteristics, but still appear technical enough to display their nonhuman characteristics [12], [13], [14]. For instance, a humanlike torso, facial social cues, and the relation between width and height of a robot head determine a robot's humanlikeness (e.g., [12], [13], [15]). The wider a robot head relative to its height, the less it is perceived as humanlike [12]. Similarly, humanlikeness ratings depend on whether participants evaluate a robot head's portrait or the holistic robot platform [15]

Such design principles were considered in the design of the social robot head "Flobi" [16]. The purpose of a social robot is to enable social human-robot interaction [17]. Therefore, Flobi has a humanlike face with eyes, eyebrows, a mouth, and a hair part. These facial features successfully evoke perceptions of emotions and robot gender [16], [18]. Simultaneously, the robot head still appears "cartoon-like" [16]. The "Meka Mobile Manipulator M1" model [19] is a service robot whose primary purpose is to assist users, for instance, in the smart home context (see [20]). Meka is a humanoid system with two dexterous arms and hands, an omnidirectional wheelbase, and an exchangeable rectangleshaped sensor head. Meka can navigate through the environment, detect and grasp objects (see [21], [22]). Quite contrary to Flobi, Meka's sensor head is wider relative to its height and does not provide any social cues to display emotions or to facilitate social communication. To take advantage of Meka's functional versus Flobi's social design, a new robot head based on Flobi's original design was developed. This new robot head, "Floka", was mounted on the Meka wheelbase to be used in the smart home context. Some features of the Flobi head had to be redesigned to match the Meka wheelbase. Compared to Flobi, Floka's head is broader, has smaller eyes, and a new hair part (see [15] for results of a study with a German sample investigating the impact of robots' body-head relation on the overall perception of Meka vs. Flobi vs. Floka).

\section{HYPOTHESES}

In the current study, we compared Japanese and German participants' evaluations of the newly designed Floka and the Meka that were intended to be used in a smart home. Due to Japanese people's general tendency to ascribe humanlike traits and habits to nonhuman entities [3], we hypothesized that Japanese participants would perceive both robots as more humanlike (H1a) and accordingly, would anthropomorphize them more by ascribing more mind to both robots (H1b) than German participants. Further, we expected that Japanese and German participants would evaluate Floka's design (H2a) and usability (H2b) more positively than Meka's.

In previous studies, Japanese participants showed more robot anxiety [7], [8] and less positive attitudes toward robots [10] than participants from Western countries. Hence,
Japanese participants were expected to show more robot anxiety (H3a), to rate both robots as uncannier (H3b), and to show less likeability (H4a), less robot attitudinal robot acceptance $(\mathrm{H} 4 \mathrm{~b})$, and less trust toward both robots $(\mathrm{H} 4 \mathrm{c})$ than German participants. Participants' tendency to anthropomorphize nonhuman agents that is inherent in Japanese culture was likely to impact participants' evaluation of the robot (see [3]). Likewise, personality traits are influenced by a culture's norms and values and therefore differ between cultures [23]. Further, for instance, agreeableness [21], extraversion, conscientiousness, neuroticism [24], and openness to experience [25] have shown to impact participants' evaluations of technology use in general and HRI in particular. The same accounted for technology commitment as another participant inherent characteristic (see [21], [26]). Consequently, to test hypotheses, individual differences in anthropomorphism, personality traits, and technology commitment were considered as covariates.

Further, because more and more service robots are developed worldwide to assist people in everyday life as personal assistants and companions [2], we explored which role Japanese and German participants would assign to the robot if they would have it at home. Related to participants' intentions to use a robot, namely, their intentional robot acceptance [27], we further explored how much they were willing to pay for the robot and for what kind of tasks they would want to use it for. Intentional robot acceptance is related to users' attitudes toward robots, namely, their attitudinal robot acceptance (see H4b). However, intentional and attitudinal robot acceptance have been proved to be distinct dimensions of robot acceptance (see [27]).

\section{METHOD}

\section{A. Design and Participants}

A total of 102 participants took part in this 2 (robot type: prototype Floka vs. Meka) x 2 (participant nationality: Japanese vs. German) between-subjects online study. 36 of the participants were Japanese (male: $n=22$, female: $n=14 ; M_{\text {age }}$ $=33.86, S D_{\text {age }}=13.19$, age range: $19-62$ years $)$ and 66 participants were German nationals (male: $n=25$, female: $n=$ $41 ; M_{\text {age }}=26.76, S D_{\text {age }}=10.15$, age range: $18-70$ years $)$. All participants reported frequent use of computers, tablet PCs, smart phones, and internet. They indicated to have had little exposure to intelligent technology, such as intelligent domestic applications, smart TV, or intelligent (virtual) assistants. Participants were naïve about the purpose of the study. Only three German participants reported that they had seen the Meka robot before. Two German participants indicated to have already known the Floka robot. The German data was previously analyzed and published in work on the impact of robots' head-body relations on participants' perceptions of Meka vs. Flobi vs. Floka [15]. Sample sizes were specified according to the heuristic that statistical requirements are fulfilled with a minimum of 20 participants per condition [28].

\section{B. Procedure}

Participants were recruited via social media and mailing lists provided by German and Japanese universities to participate in our online survey. Participants were informed that we would evaluate a newly designed robot for use in 
smart home contexts. Participants either rated Meka or Floka (see Figure 1). On top of each page of the online survey, colored photographs $(465 \mathrm{px} \times 231 \mathrm{px})$ depicted the respective robot from three perspectives (frontal, about $45^{\circ}$, and $90^{\circ}$, [15]). Questionnaire items were assessed in the same order as described in the following sections $\mathrm{C}$ and D). Further, participants indicated their experience with technology and their mood (see [21] for the impact of positive and negative affect on participants' evaluations of technology), whether they had known the robot before, and demographics like gender, age, nationality, native language, educational level, and profession.

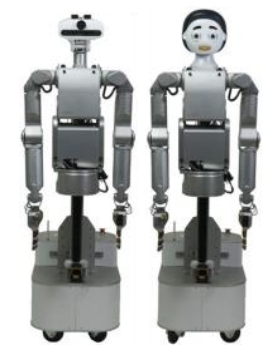

Figure 1. Meka M1 robot (left) and Floka robot (right).

\section{Dependent Measures}

Participants' responses were assessed using 7-point Likert scales (except for the two open-ended response options for the items on intentional robot acceptance, see the following section E). When necessary, items were recoded, so that high scores indicated high endorsement of the respective construct. The Japanese questionnaire was prepared and translated by a Japanese researcher.

Humanlikeness (H1a) and Anthropomorphization (H1b): One item assessed to what extent participants perceived the robot as humanlike. Mind perception is one form of humanity attribution reflecting anthropomorphization (e.g., [29], [30], [31]). 12 items [32] tapped the two dimensions of mind perception, agency ("I think the robot can make plans and work towards a goal." $, \alpha_{\text {Meka Jap }}=.71, \alpha_{\text {Floka Jap }}=.87, \alpha_{\text {Meka Ger }}=$ $76, \alpha_{\text {Floka Ger }}=.76$ ) and experience ("I think the robot can be afraid or fearful. “, $\alpha_{\text {Meka Jap }}=.55, \alpha_{\text {Floka Jap }}=.64, \alpha_{\text {Meka Ger }}=.74$, $\left.\alpha_{\text {Floka Ger }}=.82\right)$.

Design (H2a) and Usability (H2b): 15 items adapted from [33] were administered to evaluate the quality of the robot's design (e.g., "The robot's design is aesthetical.", $\alpha_{\text {Meka Jap }}=$ $\left..84, \alpha_{\text {Floka Jap }}=.79, \alpha_{\text {Meka Ger }}=.86, \alpha_{\text {Floka Ger }}=.91\right)$. To measure the robot's expected usability, we used 10 items adapted from [34] (e.g., "I think the robot would be easy to use.", $\alpha_{\text {Meka Jap }}=$ $\left..67, \alpha_{\text {Floka Jap }}=.70, \alpha_{\text {Meka Ger }}=80, \alpha_{\text {Floka Ger }}=.89\right)$.

Robot Anxiety (H3a) and Uncanniness $(\mathrm{H} 3 b)$ : Ten items [35] measured whether participants would fear to interact with the robot (e.g., "I fear, I wouldn't know how strong the robot was.", $\alpha_{\text {Meka Jap }}=.61, \alpha_{\text {Floka Jap }}=.72, \alpha_{\text {Meka Ger }}=.83, \alpha_{\text {Floka }}$ Ger $=.83)$. Three items adapted from [36] assessed whether participants perceived the robot as uncanny (e.g., "Please indicate to what extent you feel uneasy while looking at the robot." $, \alpha_{\text {Meka Jap }}=.77 \alpha_{\text {Floka Jap }}=.77, \alpha_{\text {Meka Ger }}=.91, \alpha_{\text {Floka Ger }}=$ $.90)$.

Likeability (H4a) and Attitudinal Robot Acceptance (H4b): Ten items by [37] measured the robot's likeability (e.g., "The robot is friendly.", $\alpha_{\text {Meka Jap }}=.89, \alpha_{\text {Floka Jap }}=.87$, $\left.\alpha_{\text {Meka Ger }}=.86, \alpha_{\text {Floka Ger }}=.89\right)$. We used six items to assess whether participants would accept the robot in their environment ("How much you would like to get acquainted with the robot.", $\alpha_{\text {Meka Jap }}=.89, \alpha_{\text {Floka Jap }}=.92, \alpha_{\text {Meka Ger }}=.88$, $\left.\alpha_{\text {Floka Ger }}=.84\right)$, adapted from [38].

Trust $(\mathrm{H} 4 \mathrm{c})$ : Five items adapted from [39] and [40] assessed to what extent participants indicated trust toward the robot (e.g., "I would control whether the robot completed a task properly.", $\alpha_{\text {Meka Jap }}=.57, \alpha_{\text {Floka Jap }}=.43, \alpha_{\text {Meka Ger }}=.66$, $\alpha_{\text {Floka Ger }}=.75$ ).

\section{Covariates}

Individual Differences in Anthropomorphism: Nine items from [42] were used to measure participants' tendency to anthropomorphize nonhuman entities (e.g., "Please indicate to what extent cows have intentions.", $\alpha_{\mathrm{Jap}}=.83$, $\left.\alpha_{\mathrm{Ger}}=.72\right)$.

Personality Traits: A 10-item short version (BFI-10 [43]) of the Big Five Inventory (BFI-44 [44)] assessed the five core dimensions of personality with two items per dimension: extraversion ("I am reserved.", $\alpha_{\mathrm{Jap}}=.33, \alpha_{\mathrm{Ger}}=.84$ ), agreeableness ("I am generally trusting.", $\alpha_{\mathrm{Jap}}=.26$, $\alpha_{\mathrm{Ger}}=$ .22 ), conscientiousness ("I tend to be lazy.", $\alpha_{\mathrm{Jap}}=29, \alpha_{\mathrm{Ger}}=$ .32 ), neuroticism ("I am relaxed and handle stress well.", $\alpha_{\mathrm{Jap}}$ $=64, \alpha_{\mathrm{Ger}}=.60$ ), and openness to experience ("I have few artistic interests.", $\left.\alpha_{\mathrm{Jap}}=.31, \alpha_{\mathrm{Ger}}=.55\right)$.

Technology Commitment: Three subscales [41] assessed participants' self- ratings on acceptance of new technology (e.g., "I am always interested in using new technology.", $\alpha_{\text {Jap }}$ $\left.=.79, \alpha_{\mathrm{Ger}}=.81\right)$, their competence in using technology (e.g., "To use technical devices is difficult for me. - I just can't handle it.", $\left.\alpha_{\mathrm{Jap}}=.65, \alpha_{\mathrm{Ger}}=.82\right)$, and their perceived control over technology (e.g., "I can control technical devices.", $\alpha_{\text {Jap }}$ $\left.=.73, \alpha_{\mathrm{Ger}}=.77\right)$.

\section{E. Explorative Analyses}

Role of a Robot: To further assess which role participants imagine a robot would have in their home, we used nine items proposed by [27]. Concretely, participants were instructed to imagine having the depicted robot at home. Subsequently, they had to indicate to what extent the robot would be like a domestic tool, an assistant, a friend, a human, a servant, a toy, a pet, a machine, and a colleague. [27] does not provide any information how to interpret participants' responses. However, the Ezer scale helps to explore participants' initial classification of robots.

Intentional Robot Acceptance: To measure participants' intentions to use a robot, namely their intentional robot acceptance (see [27], [45]), we used two open-response items asking participants to indicate the amount of money they would pay for the robot if they had enough money and for what task they would mainly use it for. Further, we assessed more detailed information about the task participants would mainly use the robot for. To do so, we administered five 7 point semantic differentials, participants had to indicate whether the task they had named before was safe vs. dangerous, interesting vs. boring, stereotypically female vs. stereotypically male, socially interactive vs. socially isolated, and demanding vs. simple. 


\section{RESUlTS}

\section{A. Statistical Analyses}

To test the predictions, bootstrapped multivariate analyses of covariance (MANCOVA) were performed on the dependent measures as a function of participant nationality (Japanese vs. German) and robot type (Meka vs. Floka), while technology commitment and individual differences in anthropomorphism were included as covariates. Since internal consistencies of the BFI-facets were very low [46], they were not considered as covariates. Bootstrapped analyses were needed to ensure the robustness of the statistical analyses because sample sizes were different and the minimum sample size of $\mathrm{n}=20$ participants per condition was not accomplished in the Japanese sample (see [28]).

\section{B. Main Analyses}

Humanlikeness (Hla): According to H1a, Japanese participants were expected to perceive both robot types as more humanlike than German participants. We found a statistically significant main effect of participant nationality on participants' ratings on humanlikeness, $F(1,94)=6.72, p=$ $.011, \eta_{\mathrm{p}}{ }^{2}=.067$. That means, Japanese and German participants indicated different levels of humanlikeness. Further, we found no statistically significant main effect of robot type, $F(1,94)=0.95, p=.333, \eta_{\mathrm{p}}{ }^{2}=.010$, and no statistically significant interaction effect between participant nationality and robot type, $F(1,94)=0.53, p=.467, \eta_{\mathrm{p}}{ }^{2}=.006$. This indicated that Japanese and German participants perceived Meka as equally humanlike as Floka. Means and standard deviations for humanlikeness revealed that contrary to H1a, Japanese participants rated both robot types even less humanlike than German participants, $M_{\text {Meka Jap }}=2.65, S D_{\text {Meka }}$ Jap $=1.41 ; M_{\text {Floka Jap }}=2.63 ; S D_{\text {Floka Jap }}=1.38 ; M_{\text {Meka Ger }}=3.80$, $S D_{\text {Meka Ger }}=1.52 ; M_{\text {Floka Ger }}=4.36, S D_{\text {Floka Ger }}=1.53$. Regarding the covariates' impact on ratings on humanlikeness, a statistically significant main effect of participants' tendency to anthropomorphize nonhuman agents, $F(1,94)=3.97, p=.049$, $\eta_{\mathrm{p}}{ }^{2}=.041$ on humanlikeness ratings was found. That is, participants' tendency to anthropomorphize nonhuman agents influenced their perception of humanlikeness. In line with the literature (see [3]), Japanese participants showed a statistically significantly stronger proclivity to anthropomorphize nonhuman agents, $M_{\text {Jap }}=3.17, S D_{\text {Jap }}=1.18$, than German participants, $M_{\mathrm{Ger}}=2.67, S D_{\mathrm{Ger}}=0.74, t(50.46)=-2.30, p=$ $.026, d=0.54$.

Mind Attribution (HIb): According to H1b, we predicted that Japanese participants would anthropomorphize both robot types more by ascribing more experience and more agency to them than German participants. Regarding participants' attributions of experience, a statistically significant main effect of participant nationality, $F(1,94)=5.03, p=.027, \eta_{\mathrm{p}}{ }^{2}=.051$, was obtained. That means, Japanese and German participants attributed different levels of experience to the robot they judged. Moreover, a marginally significant main effect of robot type, $F(1,94)=3.39, p=.069, \eta_{\mathrm{p}}^{2}=.035$, but no statistically significant interaction effect between participant nationality and robot type, $F(1,94)=1.60, p=.209, \eta_{\mathrm{p}}{ }^{2}=.017$ was found. Thus, Japanese and German participants tended to attribute different levels of experience to Meka than to Floka. Considering means and standard deviations, we found that Japanese participants attributed more experience to both robot types than German participants, while they tended to attribute even more experience to Floka than to Meka, $M_{\text {Meka Jap }}=2.61$, $S D_{\text {Meka Jap }}=0.91 ; M_{\text {Floka Jap }}=3.32, S D_{\text {Floka Jap }}=1.03 ; M_{\text {Meka Ger }}=$ $2.06, S D_{\text {Meka Ger }}=0.85 ; M_{\text {Floka Ger }}=2.15, S D_{\text {Floka Ger }}=0.95$. Consequently, $\mathrm{H} 1 \mathrm{~b}$ was confirmed for the experience dimension of mind. Furthermore, participants' attribution of experience was influenced by their proclivity to anthropomorphize nonhuman agents, $F(1,94)=8.37, p=.005$, $\eta_{\mathrm{p}}^{2}=.082$.

Regarding participants' attributions of agency, no statistically significant main effect of participant nationality, $F(1,94)<0.01, p=.999, \eta_{\mathrm{p}}^{2}<.001$, no statistically significant main effect of robot type, $F(1,94)=1.70, p=.196, \eta_{\mathrm{p}}{ }^{2}=.018$, and no statistically significant interaction effect between participant nationality and robot type, $F(1,94)=0.34, p=.562$, $\eta_{\mathrm{p}}{ }^{2}=.004$ emerged. This indicated that Japanese and German participants attributed the same level of agency to both robot types, $M_{\text {Meka Jap }}=3.60, S D_{\text {Meka Jap }}=0.98 ; M_{\text {Floka Jap }}=3.98$; $S D_{\text {Floka Jap }}=1.30 ; M_{\text {Meka Ger }}=3.62, S D_{\text {Meka Ger }}=1.09 ; M_{\text {Floka Ger }}=$ $3.74, S D_{\text {Floka Ger }}=1.01$. Thus, H1b was not confirmed for the agency dimension. Further, participants' attribution of agency was influenced by their proclivity to anthropomorphize nonhuman agents, $F(1,94)=7.01, p=.010, \eta_{\mathrm{p}}{ }^{2}=.069$.

Robot Design (H2a): According to H2a, we expected that Japanese and German participants would rate Floka's design more positively than Meka's design. A statistically significant main effect of participant nationality, $F(1,64)=6.88, p=.010$, $\eta_{\mathrm{p}}{ }^{2}=.068$ revealed that Japanese and German participants' ratings on robot design differed. Means and standard deviations showed that Japanese participants rated both robot types less positively on design than German participants, $M_{\text {Meka Jap }}=3.53, S D_{\text {Meka Jap }}=0.97 ; M_{\text {Floka Jap }}=3.61, S D_{\text {Floka Jap }}=$ $0.79 ; M_{\text {Meka Ger }}=3.98, S D_{\text {Meka Ger }}=0.83 ; M_{\text {Floka Ger }}=3.81$, $S D_{\text {Floka Ger }}=0.98$. A statistically nonsignificant main effect of robot type, $F(1,94)=0.17, p=.679, \eta_{\mathrm{p}}^{2}=.002$ and a statistically nonsignificant interaction effect between robot type and participant nationality, $F(1,94)=0.15, p=.699, \eta_{\mathrm{p}}{ }^{2}=$ .002 on participants' ratings on robot design indicated that Japanese and German participants rated Meka's design as positively as Floka's design. Thus, H2a was not confirmed. Participants' proclivity to anthropomorphize nonhuman agents tended to influence their ratings on robot design, $F(1,94)=$ $3.49, p=.065, \eta_{\mathrm{p}}{ }^{2}=.036$.

Robot Usability $(H 2 b)$ : We predicted that Japanese and German participants would rate Floka higher on usability than Meka (H2b). A statistically significant main effect of participant nationality, $F(1,94)=20.96, p<.001, \eta_{\mathrm{p}}{ }^{2}=.182$ revealed that Japanese and German participants indicated different levels of robot usability. Means and standard deviations revealed that Japanese participants perceived both robot types as less usable than German participants, $M_{\text {Meka Jap }}$ $=3.18, S D_{\text {Meka Jap }}=0.81 ; M_{\text {Floka Jap }}=3.59, S D_{\text {Floka Jap }}=0.80$; $M_{\text {Meka Ger }}=4.49, S D_{\text {Meka Ger }}=0.89 ; M_{\text {Floka Ger }}=4.45, S D_{\text {Floka Ger }}$ $=1.02$. A statistically nonsignificant main effect of robot type, 
$F(1,94)=1.49, p=.225, \eta_{\mathrm{p}}^{2}=.016$, and a statistically nonsignificant interaction effect between robot type and participant nationality, $F(1,94)=1.42, p=.237, \eta_{\mathrm{p}}{ }^{2}=.015$ on usability ratings showed that Meka and Floka were rated equally positively on usability. Thus, $\mathrm{H} 2 \mathrm{~b}$ was not confirmed. The covariates did not statistically significantly influence participants' usability ratings ( $p s>.05$ ).

Robot Anxiety (H3a): Furthermore, we investigated whether Japanese participants would perceive more robot anxiety than German participants (H3a). Regarding robot anxiety, there was no statistically significant main effect of participant nationality, $F(1,94)=0.46, p=.502, \eta_{\mathrm{p}}{ }^{2}=.005$. Thus, Japanese and German participants indicated the same level of robot anxiety. A statistically nonsignificant main effect of robot type, $F(1,94)=0.08, p=.783, \eta_{\mathrm{p}}{ }^{2}=.001$, and a statistically nonsignificant interaction effect between participant nationality and robot type, $F(1,94)=0.03, p=.863$, $\eta_{\mathrm{p}}{ }^{2}<.001$ revealed that Japanese and German participants indicated the same level of robot anxiety to Meka and to Floka, $M_{\text {Meka Jap }}=4.51, S D_{\text {Meka Jap }}=0.90 ; M_{\text {Floka Jap }}=4.62, S D_{\text {Floka Jap }}=$ $0.90 ; M_{\text {Meka Ger }}=3.92, S D_{\text {Meka Ger }}=1.14 ; M_{\text {Floka Ger }}=4.05$, $S D_{\text {Floka Ger }}=1.15$. Thus, H3a was not confirmed. Participants' self-rated competence in technology use influenced their ratings on robot anxiety, $F(1,94)=8.28, p=.005, \eta_{\mathrm{p}}{ }^{2}=.081$.

Uncanniness $(H 3 b)$ : Japanese participants were expected to rate both robot types as uncannier than German participants (H3b). However, no statistically significant main effect of participant nationality, $F(1,94)=0.01, p=.927, \eta_{\mathrm{p}}{ }^{2}<.001$, none of robot type, $F(1,94)=0.01, p=.917, \eta_{\mathrm{p}}{ }^{2}<.001$, and no statistically significant interaction effect between participant nationality and robot type, $F(1,94)=0.19, p=.661$, $\eta_{\mathrm{p}}{ }^{2}=.002$ were found. Thus, contrary to H3b, Japanese participants rated both robot types as uncanny as German participants, $M_{\text {Meka Jap }}=3.61, S D_{\text {Meka Jap }}=1.52 ; M_{\text {Floka Jap }}=$ $3.75, S D_{\text {Floka Jap }}=1.47 ; M_{\text {Meka Ger }}=3.10, S D_{\text {Meka Ger }}=1.58$; $M_{\text {Floka Ger }}=3.35, S D_{\text {Floka Ger }}=1.64$. Considering the covariates, a statistically significant main effect of participants' self-rated competence in technology use, $F(1,94)=3.98, p=.049, \eta_{\mathrm{p}}{ }^{2}=$ .041 and a marginally significant main effect of perceived control over technology, $F(1,94)=3.03, p=.085, \eta_{\mathrm{p}}{ }^{2}=.031$ were obtained.

Robot Likeability (H4a): We predicted that Japanese participants would like both robot types less than German participants (H4a). No statistically significant main effect of participant nationality, $F(1,94)=1.57, p=.214, \eta_{\mathrm{p}}^{2}=.016$, none of robot type, $F(1,94)=1.55, p=.216, \eta_{\mathrm{p}}{ }^{2}=.016$, and no statistically significant interaction effect between participant nationality and robot type, $F(1,94)=0.02, p=.895, \eta_{\mathrm{p}}{ }^{2}<.001$ on likeability were obtained. Hence, contrary to H4a, Japanese participants liked both robot types as much as German participants did, $M_{\text {Meka Jap }}=2.79, S D_{\text {Meka Jap }}=1.28 ; M_{\text {Floka Jap }}=$ $3.14, S D_{\text {Floka Jap }} 1.06 ; M_{\text {Meka Ger }} 2.91, S D_{\text {Meka Ger }}=1.02 ; M_{\text {Floka Ger }}$ $=3.14, S D_{\text {Floka Ger }}=1.15$. Regarding the covariates, participants' tendency to anthropomorphize nonhuman agents, $F(1,94)=4.45, p=.037, \eta_{\mathrm{p}}{ }^{2}=.045$ had an effect on their likeability ratings.

Attitudinal Robot Acceptance (H4b): According to H4b, we expected Japanese participants to indicate less attitudinal robot acceptance toward both robot types than German participants. A statistically significant main effect of participant nationality, $F(1,94)=9.39, p=.003, \eta_{\mathrm{p}}{ }^{2}=.091$ revealed that Japanese and German participants' indicated different levels of attitudinal robot acceptance. A statistically nonsignificant main effect of robot type, $F(1,94)=0.44, p=$ $.511, \eta_{\mathrm{p}}^{2}=.005$, and a statistically nonsignificant interaction effect between participant nationality and robot type, $F(1,94)$ $=0.09, p=.771, \eta_{\mathrm{p}}{ }^{2}=.001$ indicated that Japanese and German participants showed the same level of attitudinal robot acceptance toward Meka as to Floka. In line with H4b, means and standard deviations showed that Japanese participants accepted both robot types less than German participants, $M_{\text {Meka }}$ Jap $=3.55, S D_{\text {Meka Jap }}=1.55 ; M_{\text {Floka Jap }}=3.09, S D_{\text {Floka Jap }}=1.47$; $M_{\text {Meka Ger }}=3.91, S D_{\text {Meka Ger }}=1.57 ; M_{\text {Floka Ger }}=3.81, S D_{\text {Floka Ger }}$ $=1.28$. Participants' level of attitudinal robot acceptance was statistically significantly influenced by their general technology acceptance, $F(1,94)=14.08, p<.001, \eta_{\mathrm{p}}{ }^{2}=.130$. Moreover, participants' proclivity to anthropomorphize nonhuman agents had a marginally significant effect on their attitudinal robot acceptance, $F(1,94)=3.86, p=.053, \eta_{\mathrm{p}}{ }^{2}=$ .039 .

Trust $(\mathrm{H} 4 \mathrm{c}$ ): We hypothesized that Japanese participants would show less trust toward both robot types than German participants (H4c). A marginally significant main effect of participant nationality, $F(1,94)=3.49, p=.065, \eta_{\mathrm{p}}^{2}=.036$, no statistically significant main effect of robot type, $F(1,94)=$ $0.27, p=.604, \eta_{\mathrm{p}}{ }^{2}=.003$, and no statistically significant interaction effect between participant nationality and robot type, $F(1,94)=0.45, p=.506, \eta_{\mathrm{p}}{ }^{2}=.005$ were obtained on trust toward the robot. That means, in line with H3c, Japanese participants tended to indicate lower levels of trust toward both robot types, $M_{\text {Meka Jap }}=3.20, S D_{\text {Meka Jap }}=0.89 ; M_{\text {Floka Jap }}=3.44$, $S D_{\text {Floka Jap }}=0.82 ; M_{\text {Meka Ger }}=3.88, S D_{\text {Meka Ger }}=0.79 ; M_{\text {Floka Ger }}=$ $3.80, S D_{\text {Floka Ger }}=0.97$. None of the covariates had a statistically significant effect on participants' usability ratings (ps> .05).

\section{Explorative Data Analyses}

Role of a Robot: We used the Ezer scale [27] (see III. Methods, section E. Role of a Robot) to explore which role participants would assign to the robot if they would use it at home. Furthermore, we explored how much participants would be willing to pay for the robot and for what kind of task they would mainly want to use it for. The major aim of these analyses was to compare Japanese and German participants' responses. According to previous analyses, the two robot types Meka and Floka were rated similarly on all measures, except for the experience dimension of mind perception (see section B. Main Analyses). Hence, in the following analyses, we compared Japanese and German participants' responses in general, independent of robot type. Thus, we considered participant nationality as a factor, but not participants' specific evaluations of Meka and Floka, in particular. To analyze effects of nationality, independent $t$-tests were conducted to compare Japanese and German participants' responses on the respective items. As depicted in Table I, Japanese and German participants assigned similar roles to the robot they judged. They differed only in their view of the robot as a domestic tool. German participants viewed the robot they judged more as a domestic tool than Japanese participants. Descriptive statistics reveal that Japanese and German participants generally viewed 
the robot rather as a machine, a domestic tool, an assistant, a servant, and a toy than as a human, a friend, a pet, and a colleague.

TABLE I. COMPARISON OF JAPANESE AND GERMAN PARTICIPANTS' CLASSIFICATIONS OF THE ROBOTS.

\begin{tabular}{lllllll}
\hline Item & $M_{\text {Jap }}$ & $S D_{\text {Jap }}$ & $M_{\text {Ger }}$ & $S D_{\mathrm{Ger}}$ & $t$ & $p$ \\
\hline Domestic tool & 4.67 & 1.99 & 5.82 & 1.32 & 3.12 & .003 \\
Assistant & 4.53 & 1.87 & 4.76 & 1.83 & 0.60 & .550 \\
Friend & 2.22 & 1.38 & 1.94 & 1.32 & -1.02 & .311 \\
Human & 1.72 & 1.09 & 1.67 & 1.18 & -0.23 & .816 \\
Servant & 4.22 & 1.88 & 4.38 & 1.98 & 0.39 & .698 \\
Toy & 3.42 & 1.95 & 4.03 & 1.95 & 1.52 & .131 \\
Pet & 2.19 & 1.72 & 1.97 & 1.41 & -0.71 & .480 \\
Machine & 6.08 & 1.23 & 5.86 & 1.53 & -0.74 & .460 \\
Colleague & 2.19 & 1.53 & 2.45 & 1.56 & 0.81 & .420 \\
\hline
\end{tabular}

Intentional Robot Acceptance: In the following, we used two open-response items to explore how much Japanese and German participants would want to pay for the robot they judged and for what kind of task they would mainly want to use it for (see III. Methods, section E. Intentional Robot Acceptance). An independent $t$-test revealed that Japanese participants would be willing to pay statistically significantly more for the robot than German participants, $M_{\mathrm{Jap}}=7,437.13$, $S D_{\mathrm{Jap}}=14,387.61 ; M_{\mathrm{Ger}}=2279.81, S D_{\mathrm{Ger}}=3719.21, t(37.57)$ $=-2.11, p=.041, d=0.49$, (Japanese participants' responses were converted from $¥$ to $€$ ). By using 7-point semantic differentials, participants classified the task they had indicated to want to mainly use the respective robot for (e.g., $1=$ dangerous vs. $7=$ safe, see Table II). An independent $t$ test was conducted to compare Japanese and German participants' classifications of the tasks they had indicated. Japanese participants' ratings of the tasks were around scale midpoint, while compared to Japanese participants, German participants were more willing to use the respective robot for rather safe, boring, and simple tasks that do not require any social interaction with it (see Table II).

TABLE II. PARTICIPANTS' RATINGS OF THE TASK THEY WOULD MAINLY USE THE ROBOTS FOR.

\begin{tabular}{lllllll}
\hline Item & $M_{\text {Jap }}$ & $S D_{\text {Jap }}$ & $M_{\mathrm{Ger}}$ & $S D_{\mathrm{Ger}}$ & $t$ & $p$ \\
\hline Safe vs. Dangerous & 3.83 & 1.34 & 2.62 & 1.57 & -4.11 & $<.001$ \\
Interesting vs. Boring & 3.86 & 1.59 & 5.27 & 1.86 & 3.85 & $<.001$ \\
Stereotyp. female vs. Male & 4.11 & 1.43 & 3.76 & 1.24 & -1.30 & .196 \\
Interactive vs. Isolated & 3.83 & 1.63 & 4.98 & 1.78 & 3.22 & .002 \\
Demanding vs. Simple & 3.72 & 1.47 & 4.48 & 1.78 & 2.19 & .031 \\
\hline \multicolumn{7}{c}{ Note. $d f$ dangerous vs. safe $=82$, all others $d f=100}$.
\end{tabular}

\section{DISCUSSION}

In the present study, we investigated attitudes of Japanese and German participants regarding two types of service robots designed to be used in the smart home context. One was equipped with a rather "technical" sensor head, the other featured a newly designed "social" robot head. The latter one had facial social cues, such as eyes, eyebrows, and a mouth to enable social interaction [16], [17]. Furthermore, we explored which role participants generally assigned to the robot, how much they would agree to pay for it, and for what kind of task they would mainly use it for. Contrary to our predictions, Japanese participants perceived both robot types as less humanlike, but tended to attribute more experience and an equal level of agency to them compared to German participants. At the same time, Japanese participants showed a stronger proclivity to anthropomorphize nonhuman agents in general. This is in line with previous literature (see [3]). It might be grounded in the influence of Japanese Shinto religion. According to Shintoism, humanlike traits and a soul are inherent in all kind of nonhuman agents, such as gods, animals, and nature [3]. According to our results, participants' tendency to anthropomorphize nonhuman agents had a strong impact on their evaluation of the robots. As such, it affected their ratings on humanlikeness, experience, agency, robot design, likeability, and attitudinal robot acceptance. Unexpectedly, a stronger tendency to anthropomorphize nonhuman agents might have caused Japanese participants to perceive the robots even as less humanlike. In Shintoism, a soul and humanlike traits can be ascribed to any entity, even if this entity does not have a humanlike appearance (see [3]). Thus, despite a higher proclivity to anthropomorphize nonhuman entities, Japanese participants might have been well aware that the robot they judged still appeared like a technical object. However, Japanese participants' higher tendency to anthropomorphize nonhuman agents did not preclude to attribute more mind, particularly experience, to the robot. These results indicate that future research on the link between participants' cultural beliefs and their perception of robots seems promising. Additionally, Japanese participants rated the robot's design and usability less positively than German participants. In contrast to our predictions, neither Japanese, nor German participants rated the social robot Floka more positively on design and usability than the technical robot Meka. Due to the fact that both robot heads were depicted on a Meka wheelbase, participants might have perceived both robots rather as technical than as social robots. This in turn might have affected especially Japanese participants' judgments of the robot because many Japanese robots were designed after the model of Japanese anime figures. These represent social roles or traits that are commonly believed to be desirable in Japanese society. Likewise, a robot's design should reflect those desirable social roles and traits (see [47]). As predicted, Japanese participants indicated less attitudinal robot acceptance and tended to show less trust toward both robot types than German participants (see also [10], [11]). Unexpectedly, Japanese and German participants indicated the same level of robot anxiety, uncanniness, and robot likeability. Descriptive statistics revealed that Japanese and German participants rated both robot types neutral to low on all measures, such as humanlikeness, mind, robot design, usability, likeability, attitudinal robot acceptance, trust, robot anxiety and uncanniness. Likewise, exploratory analyses showed that Japanese and German participants perceived both robots as domestic tools, machines, assistants, servants, and toys rather than as human companions. Compared to Japanese participants, German participants wanted to use the robots for more safe, boring, and simple tasks that do not require any social interaction with the robot. Japanese participants were willing to pay more for the robots than German participants. This might have been influenced by the fact that living expenses are higher in Japan than in Germany [48]. Interestingly, standard deviations of the average amount of money participants indicated to be willing to pay for the robots were large in the Japanese and the German sample. This might 
indicate participants' uncertainty how to judge a robot's market value. It might also illustrate a wide range of participants' readiness to pay for a robot.

Both the German and the Japanese sample were relatively heterogeneous regarding age and socio-economic status. Japanese participants were on average slightly older and indicated a lower educational level than German participants. Although our sample covered a wide age range, the sample sizes between Japanese and German participants were not balanced. Both Japanese and German participants indicated to be familiar with internet, computer, and smart phone use, but not with intelligent agents. Moreover, especially naïve Japanese participants reported problems completing the study as they felt they lacked knowledge of robots and thus did not feel competent to evaluate a robot's design. Similarly, in a HRI study situated in an intelligent robotics apartment, [49] found that participants were not willing to interact with a robot because they did not feel competent to judge its capabilities. This previous research revealed participants' need for detailed information about a robot's functions before HRI could successfully be initiated. A lack of information and experience with robots might cause feelings of insecurity (see [49]). To feel safe, people want to control a technical system such as a robot any time (see [40], [49]).

\section{A. Conclusion}

The objection, especially by Japanese participants, not feeling competent to judge a robot due to a lack of knowledge of robots underlines the results of the current study. These indicated that statements such as "People just can't wait to have a robot at home" and "Especially Japanese people are crazy about robots", as well as the common belief that Japanese people are more familiar with robots than people in Western cultures (e.g., [1]) might be misleading. According to our empirical data, the reality seems to be that Japanese and German participants have similar, not very enthusiastic views on robots (see also [50] for findings on educational robots). The gap between Japanese and German nationals' opinions about robots seems not as pronounced as previous literature has suggested (e.g., [7], [8], [10]). According to our data, neither German, nor Japanese participants reported having been exposed to robots as much as would stereotypically be expected. Obviously, Japanese and German participants did not see any benefit from using robots and avoided to initiate direct human-robot interaction. To motivate people to interact with robots in their daily life, the benefit of using a robot should be obvious, while the robot should appear predictable and thus easy to control (e.g., [40], [45], [49]). This to be realized, user preferences, attitudes, and concerns about robots need to be considered and investigated. We recommend developing robot platforms based on empirical user studies that highlight user preferences and psychological aspects underlying a successful and positive HRI rather than relying on stereotypical social robot roles users might consider as appropriate. Moreover, the benefit from using a robot needs to be communicated in a way users understand and feel concerned while giving detailed information about a robot's functions and abilities (see also [49]). To create a positive HRI, the users' perspective should be taken into sharper focus to gain a more realistic view on robots and their potential users.

\section{ACKNOWLEDGMENT}

The authors thank all members of the Junior Expert Workshop 2017 organized by the Japanese-German Center Berlin for their kind support with data collection in Japan. A special thank goes to Yuri Seritas for kindly translating the questionnaire to Japanese language. We report all data exclusions (if any), all manipulations, all measures in the study, and how sample sizes were calculated.

\section{REFERENCES}

[1] T. N. Hornyak, Loving the machine: The art and science of Japanese robots. Tokyo: Kodansha International, 2006.

[2] International Federation of Robotics (IFR), "Why service robots are booming worldwide,", IFR Press Releases, 2017, retrieved from: https://ifr.org/ifr-press-releases/news/why-service-robots-arebooming-worldwide.

[3] J. Hackin, Asiatic mythology: a detailed description and explanation of the mythologies of all the great nations of Asia. Asian Educational Services, 1932.

[4] H. Kamide, F. Eyssel, and T. Arai, "Psychological anthropomorphism of robots," Int. Conf. Soc. Rob, in Lect. Notes Comput. Sci., Springer, 2013, pp. 199-208.

[5] N. Epley, A. Waytz, and J. T. Cacioppo, "On seeing human: a threefactor theory of anthropomorphism." Psychol. Rev., 2007, vol. 114, no. 4 , pp. $864-886$.

[6] K. S. Haring, D. Silvera-Tawil, T. Takahashi, M. Velonaki, and K. Watanabe, "Perception of a humanoid robot: a cross-cultural comparison," in Proc. IEEE Int. Symp. Rob. Hum. Interact. Commun. Ro-Man, 2015, pp. 821-826.

[7] C. Bartneck, T. Nomura, T. Kanda, T. Suzuki, and K. Kennsuke, "A cross-cultural study on attitudes towards robots," in Proc. HCI Int., 2005, pp. 1-3.

[8] C. Bartneck, T. Suzuki, T. Kanda, and T. Nomura, "The influence of people's culture and prior experiences with Aibo on their attitude towards robots," AI Soc.., 2007, vol. 21, no. 1-2, pp. 217-230.

[9] H. Kamide and T. Arai, "Perceived comfortableness of anthropomorphized robots in us and Japan," Int. J. Soc. Rob., 2017, vol. 9, pp. 537-543.

[10] C. Bartneck, "Who like androids more: Japanese or US Americans?", in Proc. IEEE Int. Symp. Rob. Hum. Interact. Commun. Ro-Man, 2008, pp. 553-557.

[11] K. S. Haring, D. Silvera-Tawil, Y. Matsumoto, M. Velonaki, and K. Watanabe, "Perception of an android robot in Japan and Australia: A cross-cultural comparison," in Lect. Notes Artif. Intell., Springer, 2014, vol. 8755, pp. 166-175.

[12] C. F. Disalvo, F. Gemperle, J. Forlizzi, and S. Kiesler, “All robots are not created equal: The design and perception of humanoid robot heads, in Proc. Conf. Des. Interact. Syst., ACM, 2002, pp. 321-326.

[13] B. R. Duffy, "Anthropomorphism and the social robot," Rob. Auton. Syst., 2003, vol. 42, no. 3-4, pp. 177-190.

[14] M. Mori, "The uncanny valley," Energy, 1970, vol. 7, no. 4, pp. 3335 .

[15] J. Bernotat and F. Eyssel, "An evaluation study of robot designs for smart environments," in Proc. Comp. ACM/IEEE Int. Conf. Hum. Rob. Interact., ACM, 2017, pp. 87-88.

[16] F. Hegel, F. Eyssel, and B. Wrede, "The social robot Flobi: Key concepts of industrial design," in Proc. IEEE Int. Symp. Rob. Hum. Interact. Commun. Ro-Man, 2010, pp. 107-112.

[17] F. Hegel, C. Muhl, B. Wrede, M. Hielscher-Fastabend, and G. Sagerer, "Understanding social robots," IEEE in Adv. Comp. Hum. Interact., Int. Conf., 2009, pp. 169-174.

[18] F. Eyssel and F. Hegel, "(s) he's got the look: Gender stereotyping of robots," J. Appl. Soc. Psychol., 2012, vol. 42, no. 9, pp. 2213-2230.

[19] J. Weber, A. Edsinger, and A. Metzger, "Meka robotics," 2006, San Francisco.

[20] S. M. zu Borgsen, T. Korthals, F. Lier, and S. Wachsmuth, "ToBI team of Bielefeld: Enhancing robot behaviors and the role of multirobotics in robocup@home robocup," Int. Symposium, Lect. Notes Comput. Sci., 2017, vol. 9776, p. 577-588. 
[21] J. Bernotat and F. Eyssel, "A robot at home- - How affect, technology commitment, and personality traits influence user experience in an intelligent robotics apartment," in Proc. IEEE Int. Symp. Rob. Hum. Interact. Commun. Ro-Man, 2017, pp. 641-646.

[22] S. M. zu Borgsen, J. Bernotat, and S. Wachsmuth, "Hand in hand with robots: Differences between experienced and naïve users in humanrobot handover scenarios," Int. Conf. Soc. Rob, in Lect. Notes Comput. Sci., Springer, 2017, pp. 587-596.

[23] G. Hofstede and R. R. McCrae, "Personality and culture revisited: Linking traits and dimensions of culture," Cross-cultural research, 2004, vol. 38, no. 1, pp. 52-88.

[24] T. Barnett, A. W. Pearson and F. W. Kellermans, "Five-factor model personality traits as predictors," Eur. J. Inf. Syst., 2015, vol. 24, no. 4, pp. 374-390. doi:10.1057//ejis.2014.10

[25] S. Devaraj, R. F. Easley, and J. M. Crant, "Research note-How does personality matter? Relating the five-factor model to technology acceptance and use," Inf. Syst. Res., 2008, vol. 19, no. 1, pp. 93-105.

[26] D. R. Compeau and C. A. Higgins, "Computer self-efficacy: Development of a measure and initial test," MIS Quarterly, 1995, vol 19, no. 2, 189-211.

[27] N. Ezer, "Is a robot an appliance, teammate, or friend? Age-related differences in expectations of and attitudes towards personal homebased robots (Unpublished work style)" Georgia Institute of Technology, 2008, unpublished.

[28] A. Field, Discovering statistics using IBM SPSS statistics, Sage, 2013.

[29] F. Eyssel and D. Kuchenbrandt, "Social categorization of social robots: Anthropomorphism as a function of robot group membership," Br. J. Soc. Psychol., 2012, vol. 51, no. 4, pp. 724-731.

[30] F. Eyssel and S. Loughnan, "It don't matter if you're black or white, Int. Conf. Soc. Rob, in Lect. Notes Comput. Sci., Springer, 2013, pp. 422-431.

[31] F. Eyssel, R. Wullenkord, and V. Nitsch, "The role of self-disclosure in human-robot-interaction," in Proc. IEEE Int. Symp. Rob. Hum. Interact. Commun. Ro-Man, 2017, pp. 922-927.

[32] H. M. Gray, K. Gray, and D. M. Wegner, "Dimensions of mind perception," Science, 2007, vol. 315, no. 5812, p. 619.

[33] M. Hassenzahl, "User experience (UX): towards an experiential perspective on product quality," in Proc. Conf. Interact. Hum. Mach., ACM, 2008, pp. 11-15.

[34] J. Brooke, "SUS - A quick and dirty usability scale," Usab. Eval. Indust., 1996, vol. 189, no. 194, pp. 4-7.

[35] T. Nomura, T. Kanda, T. Suzuki, and K. Kato, "Prediction of human behavior in human-robot interaction using psychological scales for anxiety and negative attitudes toward robots," IEEE Trans. Rob., 2008, vol. 24, no. 2, pp. 442-451.

[36] K. Gray and D. M. Wegner, "Feeling robots and human zombies: Mind perception and the uncanny valley," Cognition, 2012, vol. 125, no. 1 , pp. $125-130$.

[37] S. Reysen, "Construction of a new scale: The Reysen likability scale," Soc. Behav. Pers.: Int. J., 2005, vol. 33, no. 2, pp. 201-208.

[38] F. Eyssel, D. Kuchenbrandt, S. Bobinger, L. de Ruiter, F. Hegel, H. Yanco, V. Evers, A. Steinfeld, and O. C. Jenkins, "'If you sound like me, you must be more human': On the interplay of robot and user features on human-robot acceptance and anthropomorphism," in Proc. Comp. ACM/IEEE Int. Conf. Hum. Rob. Interact., ACM, 2012, 125126.

[39] A. Waytz, J. Heafner, and N. Epley, "The mind in the machine: Anthropomorphism increases trust in an autonomous vehicle," J. Exp. Soc. Psychol., 2014, 52, 113-117.

[40] B. Schiffhauer, J. Bernotat, F. Eyssel, R. Bröhl, and J. Adriaans, "Let the user decide! user preferences regarding functions, apps, and control modalities of a smart apartment and a service robot," in Lect. Notes Artif. Intell., Springer, 2016, vol. 9799, pp. 971-981.

[41] F. J. Neyer, J. Felber, and C. Gebhardt, "Entwicklung und Validierung einer Kurzskala zur Erfassung von Technikbereitschaft," [Development and validation of a brief measure of technology commitment], Diagnostica, 2012, 58, 2, 87-99.

[42] A. Waytz, N. Epley, and J. T. Cacioppo, "Social cognition unbound: Insights into anthropomorphism and dehumanization," Curr. Dir. Psychol. Sci., 2007, vol. 19, no. 1, pp. 58-62.
[43] B. Rammstedt and O. P. John, "Measuring personality in one minute or less: A 10-item short version of the big five inventory in English and German," J. Res. Pers., 2007, vol. 41, no. 1, pp. 203-212.

[44] P. T. Costa and R. R. McCrae, "Normal personality assessment in clinical practice: The neo personality inventory." Psychol. Asses. 1992 , vol. 4, no. 1, p. 5-13.

[45] F. D. Davis, R. P. Bagozzi, and P. R. Warshaw, "User acceptance of computer technology: A comparison of two theoretical models," Manage. Sci., 1989, vol. 35, no. 8, pp. 982-1003.

[46] N. Schmitt, "Uses and abuses of coefficient alpha." Psychol. Assess., 1996, vol. 8, no. 4, p. 350-353.

[47] S. Šabanović, "Inventing Japan's 'robotics culture': The repeated assembly of science, technology, and culture in social robotics." Soc. Stud. Sci., 2014, vol. 44, no. 3, pp. 342-367.

[48] Federal Statistical Office, 2018, retrieved from: https://www.destatis.de/DE/PresseService/Presse/Pressemitteilungen/2 014/05/PD14_157_61621.html, 2018.

[49] J. Bernotat, B. Schiffhauer, F. Eyssel, P. Holthaus, C. Leichsenring, V. Richter, M. Pohling, B. Carlmeyer, N. Köster, S. M. zu Borgsen et al., "Welcome to the future-How naive users intuitively address an intelligent robotics apartment," in Lect. Notes Artif. Intell., Springer, 2016, vol. 9979, pp. 982-992.

[50] N. Reich-Stiebert and F. Eyssel, "Learning with educational companion robots? Toward attitudes on education robots, predictors of attitudes, and application potentials for education robots," Int. J. Soc. Rob., 2015, vol. 7, no. 5, pp. 875-888. 